DOI: https://doi.org/10.46296/ig.v1i2.0003

\title{
OBTENCIÓN DE VINAGRE DE VINO A PARTIR DEL MUCÍLAGO Y EXUDADO DE CACAO CRIOLLO (THEOBROMA CACAO L.)
}

\section{OBTAINING OF WINE VINEGAR FROM THE MUCÍLAGO AND EXUDED FROM CREOLE COCOA (THEOBROMA CACAO L.)}

\author{
Vera-Loor José Edwin ${ }^{1 *}$; Mera-Vélez Sabina Andrea2; Morales-Paredes Carlos Augusto3; \\ Loor-Vélez Joselyn Dayanari ${ }^{4}$ \\ ${ }^{1}$ Universidad Técnica de Manabí, UTM. Portoviejo, Ecuador. \\ ${ }^{1}$ Universidad Técnica de Manabí, UTM. Portoviejo, Ecuador. \\ ${ }^{3}$ Universidad Laica Eloy Alfaro de Manabí, ULEAM. Manta, Ecuador. \\ ${ }^{4}$ Universidad Técnica de Manabí, UTM. Portoviejo, Ecuador.
}

*Correo: josveraloor@gmail.com

\begin{abstract}
Resumen
El presente trabajo se basa en la obtención de vinagre de vino a partir de los residuos de la fermentación espontánea del cacao, mediante dos procesos microbianos separados; siendo primero una fermentación alcohólica de los azúcares naturales presentes en el mucílago de cacao, en un rango de 10 a 15\%, adicionando sacarosa para rectificar el contenido de sólidos solubles para la acción y conversión del género Saccharomyces Cerevisiae, y en segundo lugar, la fermentación oxidativa del alcohol obtenido; para esto se realizó la caracterización química del sustrato, estableciendo las condiciones óptimas para una fermentación acética con ayuda de las bacterias ácido acéticas. Se llevó a cabo un diseño experimental por triplicado a diferentes concentraciones $(0,11$; $0,16 ; 0,20) \mathrm{g} / \mathrm{L}$ cada una con y sin administración de fuente de nitrógeno, obteniendo en la combinación A1B1 mayor reducción de grados Brix. Se diseñó un biorreactor a escala de laboratorio con inyección de oxígeno (proveniente de una bomba de aire) y temperatura ambiente, empleando una madre de vinagre obtenida a partir de las bacterias nativas del mucílago y exudado de cacao, de esta forma se obtuvo un vinagre de vino de cacao con una composición de 18,8 g de ácido acético/L en un tiempo de 108 horas de fermentación.
\end{abstract}

Palabras clave: cacao criollo, exudado, mucílago, Theobroma Cacao L., vinagre, vino.

\begin{abstract}
The present work is based on obtaining wine vinegar from the capture of the residues resulting from the spontaneous fermentation of cocoa, through two separate microbial processes; being first an alcoholic fermentation of the natural sugars present in the cocoa mucilage, in a range of 10 to $15 \%$, adding sucrose to rectify the soluble solids content for the action and conversion of the genus Saccharomyces Cerevisiae, and secondly the fermentation oxidative of the alcohol obtained, for this the chemical characterization of the substrate was carried out, establishing the optimal conditions for acetic fermentation with the help of acetic acid bacteria. An experimental design was carried out in triplicate at different concentrations $(0.11 ; 0.16 ; 0.20) \mathrm{g} / \mathrm{L}$ each with and without administration of a nitrogen source, obtaining in the A1B1 combination a greater reduction in Brix degrees, based on this, a laboratory-scale bioreactor with oxygen injection (from an air pump) and room temperature was designed, using a vinegar stem obtained from the native bacteria of the mucilage and cocoa exudate, in this way a cocoa wine vinegar was obtained with results of $18.8 \mathrm{~g}$ of acetic acid / $\mathrm{L}$ in a time of 108 hours of fermentation.
\end{abstract}

Keywords: Creole cocoa, exudate, mucilage, Theobroma Cacao L, vinegar, wine.

Información del manuscrito:

Fecha de recepción: 29 de marzo de 2018

Fecha de aceptación: 18 de mayo de 2018

Fecha de publicación: 10 de julio de 2018 


\section{Introducción}

Según Estrella (2013) el mucílago provee las condiciones adecuadas para el proceso de fermentación y para la formación de las sustancias precursoras del sabor y aroma, además con su extracto líquido se pueden elaborar mermeladas y otros productos. Asimismo, Braudeau (2001) indica que la pulpa cuyo $\mathrm{pH}$ es ácido se asocia a la presencia de ácido cítrico, lo cual constituye un medio favorable para las levaduras, por lo que su contaminación microbiológica se inicia rápidamente una vez que las habas han sido extraídas de las mazorcas, ya sea por el simple contacto con las manos de los trabajadores o con el material utilizado para el transporte y el tratamiento del cacao, esto debido a los insectos atraídos por el mucílago azucarado.

El vinagre (del latín "vinum acre», «vino agrio») es un líquido miscible en agua, con sabor agrio. Este se obtiene mediante dos procesos microbiológicos independientes: (i) fermentación alcohólica de los azúcares naturales presentes en el mucílago de cacao y (ii) fermentación oxidativa del alcohol obtenido. Aunque el mucílago es necesario para la fermentación, a menudo hay más de lo necesario. Actualmente se sabe que a nivel comercial se aprovecha sólo la almendra, la cual representa un 10\% de la masa del fruto fresco, siendo sus residuos implementados como abono. A partir de lo expuesto se realizó el diseño de un proceso para obtener vinagre de vino de los residuos resultantes de la fermentación espontánea del cacao, específicamente de mucílago y exudado.

\section{Metodología}

El proceso para obtener el vinagre de cacao parte de la fermentación oxidativa del vino, es decir, acondicionando el sustrato alcohólico con la inoculación de un cultivo madre para su posterior fermentación acética. Durante cada etapa del proceso se tomaron muestras para su determinación analítica y así tener un control sobre el producto obtenido.

\subsection{Recepción de materia prima}

Para este estudio se realizó la captación de materia prima (Cacao Theobroma L.) de las zonas aledañas al cantón Portoviejo, Manabí, Ecuador, seleccionando el 
mucílago y exudado más claro, limpio y libre de impurezas. Además de la adquisición de mazorcas de cacao para constatar la distribución porcentual másica.

\subsubsection{Diseño del reactor batch a escala de laboratorio}

Se implementaron botellas de vidrio de $600 \mathrm{~mL}$ de capacidad con acoplamiento de una válvula de aireación manipulable, facilitando el paso de fermentación alcohólica a oxidativa sin necesidad de cambiar de reactor. La bomba permite que el aire ingrese de forma continua, regulada y en exceso, puesto que se debe considerar la solubilidad del oxígeno, así como la formación de burbujas, por lo cual el exceso de oxígeno es necesario. Por otra parte, se cuantificó la producción de $\mathrm{CO}_{2}$ mediante una trampa de $\mathrm{NaOH}$.

\subsubsection{Cultivo madre como inóculo}

En la obtención del cultivo madre como inóculo, el exudado se deja en reposo en un recipiente con amplia superficie de contacto para la oxigenación y tapado con gasas para evitar la contaminación física del cultivo, el cual tiene varios días de fermentación natural en la cacaotera, para que, al cabo de aproximadamente 6 días, las bacterias produzcan una película celulosa en la superficie. Debido al bajo contenido de alcohol $\left(2^{\circ} \mathrm{GL}\right)$, se añade $1 \mathrm{~mL}$ de etanol al $94 \%$ para mantener en condiciones adecuadas a las bacterias acéticas.

\subsection{Diseño experimental}

El vinagre de vino se obtuvo mediante dos procesos microbianos separados; (i) fermentación alcohólica de los azúcares naturales presentes en el mucílago de cacao, en un rango de al menos 10 a $15 \%$ adicionando sacarosa para rectificar el contenido de sólidos solubles para la acción y conversión del género Saccharomyces Cerevisiae, la levadura empleada debe activarse con azúcar y temperatura $\left(35^{\circ} \mathrm{C}\right)$, durante 15 minutos. (ii) $\mathrm{La}$ fermentación oxidativa del alcohol obtenido, requiere de una caracterización química del sustrato, estableciendo las condiciones óptimas para una fermentación acética con ayuda de las bacterias ácido acéticas. Se determinó el requerimiento de oxígeno mínimo a partir del cálculo de la demanda teórica, además de la inoculación en el reactor con $5 \mathrm{~mL}$ del cultivo madre. 


\subsection{Medición de variables experimentales}

Para la inspección y acondicionamiento de la materia prima, evaluaciones intermedias del proceso y verificación de la calidad del producto se aplicaron las siguientes técnicas.

Tabla 1.

Ensayos y técnicas de control empleadas en la presente investigación

\begin{tabular}{|c|c|}
\hline Ensayos / Técnicas & Referencia \\
\hline $\begin{array}{l}\text { Acidez Total, (como } \\
\text { ácido acético) \% m/v }\end{array}$ & $\begin{array}{l}\text { AOAC }^{*} 930.35 \\
\text { INEN } 341\end{array}$ \\
\hline $\begin{array}{l}\text { Acidez fija, (como ácido } \\
\text { acético), \% m/v }\end{array}$ & $\mathrm{AOAC}^{*} 930.35$ \\
\hline $\begin{array}{l}\text { Acidez volátil, (como } \\
\text { ácido acético), \% m/v }\end{array}$ & $A O A C^{*} 930.35$ \\
\hline $\begin{array}{l}\text { Alcohol etílico a } 20^{\circ} \mathrm{C} \text {, } \\
\% \mathrm{v} / \mathrm{v}\end{array}$ & $\begin{array}{l}\text { AOAC }^{*} 930.35 \\
\text { INEN } 360\end{array}$ \\
\hline $\begin{array}{l}\text { Potencial de Hidrógeno, } \\
\mathrm{pH} \text { a } 20^{\circ} \mathrm{C}\end{array}$ & $\mathrm{AOAC}^{*} 981.12$ \\
\hline Determinación de ํㅏix & PCE Instruments \\
\hline Test de Fehling & $\begin{array}{l}\text { AOAC, 1965, p. } \\
495\end{array}$ \\
\hline $\begin{array}{l}\text { Recuento de células en } \\
\text { un líquido }\end{array}$ & $\begin{array}{l}\text { Cámara de } \\
\text { Neubauer }\end{array}$ \\
\hline $\begin{array}{l}\text { Clarificación de mostos } \\
\text { y vinos. }\end{array}$ & Molina, 2000 \\
\hline
\end{tabular}

* Feldsine et al., (2002)

\subsubsection{Medición de rendimiento}

Los sistemas de fermentación originaron un cálculo del rendimiento real de este proceso, comparando la concentración total del vinagre descargado con la concentración total de la materia prima. En ambos casos, la concentración total es la suma de la acidez total (expresada en $g$ de ácido acético/100 mL) y de la acidez que se espera obtener por transformación de alcohol presente. Introduciendo un factor de corrección para compensar las distintas formas de expresar las concentraciones de ambos productos (Llaguno \& Polo, 1991).

\section{Resultados y discusión}

\subsection{Concentración del cacao}

Los resultados obtenidos en la extracción del mucílago del fruto de cacao y sus partes constitutivas demuestran que, al incorporar estas materias primas no convencionales, es decir aprovechando un $24,48 \%$ de los residuos que representaron el $85 \%$ del fruto, el cacao es aprovechado en un $21 \%$ más. Estos datos se reflejan en la tabla 2 . 
Tabla 2.

Pesos promedios del fruto de cacao y sus partes constitutivas

\begin{tabular}{|c|c|c|c|c|c|c|} 
Muestras & $\begin{array}{c}\text { Peso } \\
\text { neto } \\
\text { (lb) }\end{array}$ & $\begin{array}{c}\text { Cascara } \\
\text { pura } \\
\mathbf{( l b )}\end{array}$ & $\begin{array}{c}\text { Mucilago } \\
\mathbf{y} \text { tripas } \\
\mathbf{( l b )}\end{array}$ & $\begin{array}{c}\text { Pepas } \\
\text { húmedas } \\
\mathbf{( l b )}\end{array}$ & $\begin{array}{c}\text { Pepas } \\
\text { secas } \\
\mathbf{( l b )}\end{array}$ & $\begin{array}{c}\text { Exudado } \\
\text { (lb) }\end{array}$ \\
\hline Cacao 1 & 3 & 2,475 & 0,375 & 0,15 & 0,12 & 0,03 \\
\hline Cacao 2 & 2 & 1,25 & 0,238 & 0,512 & 0,35 & 0,162 \\
\hline Cacao 3 & 1,75 & 0,9 & 0,509 & 0,341 & 0,32 & 0,021 \\
\hline Cacao 4 & 1,11 & 0,6 & 0,31 & 0,2 & 0,192 & 0,008 \\
\hline Cacao 5 & 1,5 & 0,8 & 0,279 & 0,421 & 0,4 & 0,021 \\
\hline Total & $\mathbf{9 , 3 6}$ & $\mathbf{6 , 0 2 5}$ & $\mathbf{1 , 7 1 1}$ & $\mathbf{1 , 6 2 4}$ & $\mathbf{1 , 3 8 2}$ & $\mathbf{0 , 2 4 2}$ \\
\hline \% & $\mathbf{1 0 0}$ & $\mathbf{6 4}$ & $\mathbf{1 8}$ & $\mathbf{1 7}$ & $\mathbf{1 5}$ & $\mathbf{3}$ \\
\hline
\end{tabular}

3.2. Estudio de la fermentación alcohólica

\subsubsection{Análisis y descripción del} proceso de fermentación alcohólica

Para mejorar las condiciones $y$ reducir el tiempo de la fermentación se evalúa la mejor combinación sustrato-MO-fuente de nitrógeno, valorando la fermentación con 3 concentraciones de Saccharomyces cerevisiae con ausencia y presencia de sulfato de amonio por triplicado como se muestra en la tabla 3 , dando un total de 18 unidades experimentales que fueron evaluadas en función de la reducción de los ${ }^{\circ}$ Brix, con un $\mathrm{pH}$ controlado de 4,5 y a temperatura ambiente.

\section{Tabla 3.}

Combinaciones experimentales con su respectiva codificación

\begin{tabular}{|c|c|c|c|c|c|c|}
\hline Condiciones de Estudio & A1B1 & A1B2 & A1B3 & A2B2 & A2B2 & A2B3 \\
\hline *Saccharomyces Cerevisiae, g/L & 0,20 & 0,16 & 0,11 & 0,20 & 0,16 & 0,11 \\
\hline Sulfato de amonio, g/L & 0,1 & 0,1 & 0,1 & 0 & 0 & 0 \\
\hline Potencial de Hidrógeno, UpH & 4,5 & 4,5 & 4,5 & 4,5 & 4,5 & 4,5 \\
\hline Temperatura, ${ }^{\circ} \mathrm{C}$ & 35 & 35 & 35 & 35 & 35 & 35 \\
\hline
\end{tabular}


En la tabla 4 se muestra que la combinación A1B1 $(0,1 \mathrm{~g} / \mathrm{L}$ de $\left(\mathrm{NH}_{4}\right)_{2} \mathrm{SO}_{4} \quad$ y $\quad 0,11 \mathrm{~g} / \mathrm{L}$ Saccharomyces cerevisiae) es el resultado más adecuado de la experimentación del proceso de fermentación alcohólica, debido a una mayor reducción de los Brix, desde 11 a 7,2; además de tener una menor desviación estándar, por lo tanto, se define como la combinación utilizada en la elaboración del vino de cacao (posterior sustrato para la fermentación acética).

\section{Tabla 4.}

Resultados del diseño experimental de las diferentes concentraciones de levaduras y nutrientes

\begin{tabular}{|c|c|c|c|c|}
\hline Combinación & Réplicas & $\begin{array}{c}{ }^{\circ} \text { Brix } \\
\text { Inicial }\end{array}$ & $\begin{array}{l}{ }^{\circ} \text { Brix } \\
\text { Final }\end{array}$ & $\begin{array}{c}\text { Desviación } \\
\text { estándar }\end{array}$ \\
\hline \multirow{3}{*}{ A1B1 } & I & 11 & 7.2 & \multirow{3}{*}{0.12} \\
\hline & II & 11 & 7.2 & \\
\hline & III & 11 & 7 & \\
\hline \multirow[t]{3}{*}{ A1B2 } & 1 & 11 & 8 & \multirow{3}{*}{0.69} \\
\hline & II & 11 & 8 & \\
\hline & III & 11 & 9.2 & \\
\hline \multirow{3}{*}{ A1B3 } & 1 & 11 & 8.8 & \multirow{3}{*}{0.20} \\
\hline & II & 11 & 8.4 & \\
\hline & III & 11 & 8.6 & \\
\hline \multirow{3}{*}{ A2B1 } & 1 & 11 & 9.8 & \multirow{3}{*}{0.53} \\
\hline & II & 11 & 9 & \\
\hline & III & 11 & 10 & \\
\hline \multirow{3}{*}{ A2B2 } & I & 11 & 9.4 & \multirow{3}{*}{0.31} \\
\hline & II & 11 & 9 & \\
\hline & III & 11 & 9.6 & \\
\hline \multirow{3}{*}{ A2B3 } & I & 11 & 9.4 & \multirow{3}{*}{0.23} \\
\hline & II & 11 & 9.4 & \\
\hline & III & 11 & 9.8 & \\
\hline
\end{tabular}

La fermentación alcohólica del sustrato de mucílago y exudado de cacao fue realizada bajo las mismas condiciones establecidas en la investigación de Villagómez (2013), variando características fisicoquímicas como la materia prima (cacao criollo), Brix inicial, acidez inicial y $\mathrm{pH}$ inicial.

Se puede observar en la figura 1, el consumo de los azúcares reportado durante la fermentación alcohólica, no obstante, en esta curva se observa el consumo del sustrato corregido a 17 Brix, apreciándose en la gráfica la existencia de 2 diauxias, lo que sugiere que las bacterias desdoblaron dos tipos de azúcares presentes en el mucílago, diferentes a la sacarosa; el mosto poseía inicialmente 11,8 Brix, y es justamente en el rango 11-12 Brix donde se denota irregularidad en la curva, eventualmente disminuye la tasa de consumo del sustrato, reduciendo finalmente su acción a $7,8^{\circ}$ Brix en un tiempo de 236 horas. 


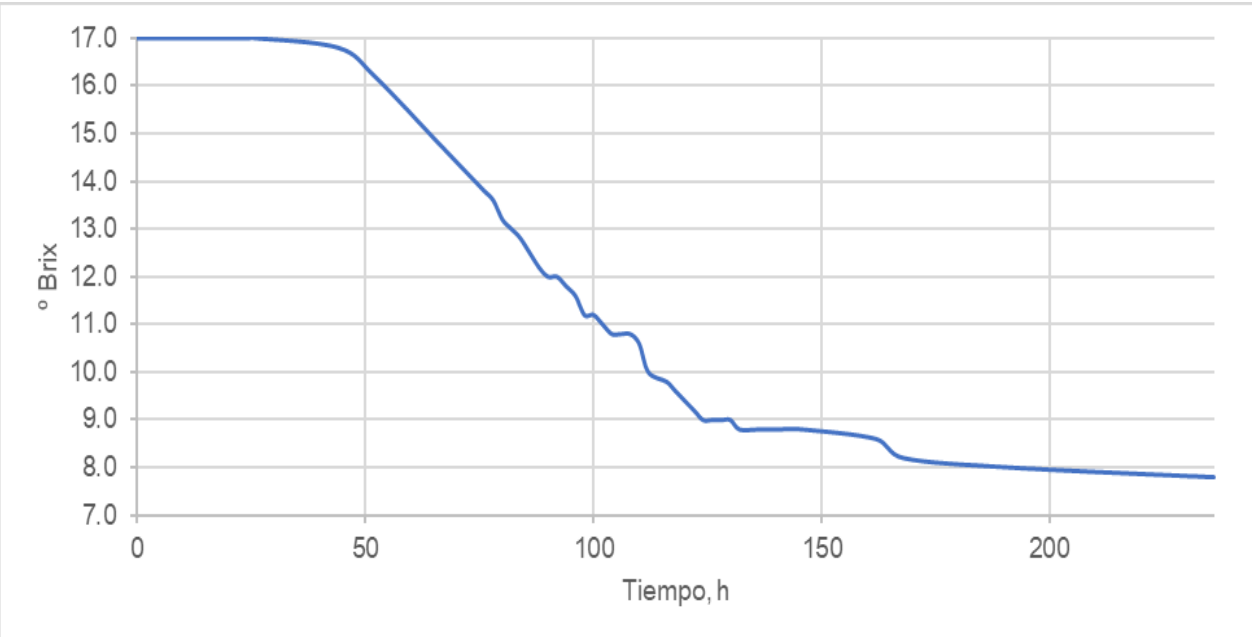

Figura 1. Cinética de fermentación alcohólica del mucílago y exudado del cacao criollo

Por otra parte en la figura 2 se puede observar el comportamiento de las levaduras Saccharomyces Cerevisiae frente al sustrato durante la fermentación alcohólica, en donde se identificó la fase de latencia (aclimatación y adaptación de los microorganismos) comprendida ente las horas: 0-44; la fase exponencial (reproducción y crecimiento de los microorganismos) oscila entre las 44-124 horas, siendo la etapa en la que se alcanza el mayor consumo de los sustratos por las levaduras antes de llegar a la fase estacionaria, donde los microorganismos mantienen un consumo mínimo antes de llegar a la muerte microbiana.

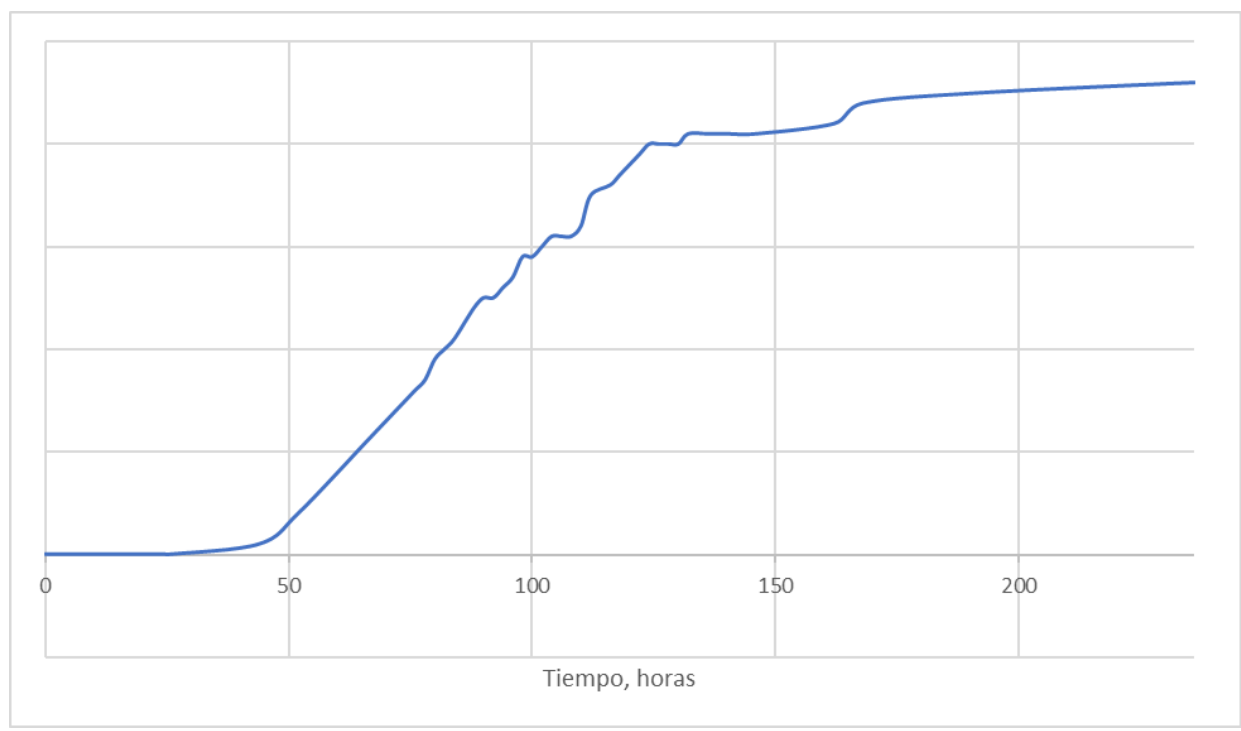

Figura 2. Cinética del crecimiento de la levadura Saccharomyces Cerevisiae durante la fermentación alcohólica 


\subsection{Coeficientes estequiométricos} y rendimientos máximos

En la tabla 5 y 6 se pueden observar los cálculos estequiométricos y los rendimientos teóricos y máximos termodinámicos, obtenidos a partir de la biomasa del proceso experimental de la fermentación alcohólica y acética. Sin embargo, en comparación con el rendimiento de producto (Yps) la reacción favoreció a la producción de biomasa, esto debido a la intervención de varios factores que influyen en el rendimiento de la biomasa, incluyendo la composición del medio y la naturaleza de las fuentes de nitrógeno y carbono, $\mathrm{pH}$ y temperatura.

Tabla 5.

Rendimientos experimentales y máximos del proceso de fermentación alcohólica

\begin{tabular}{|c|c|c|c|c|}
\hline \multicolumn{5}{|c|}{$\mathrm{C}_{12} \mathrm{H}_{22} \mathrm{O}_{11}+\left(\mathrm{NH}_{4}\right)_{2} \mathrm{SO}_{4} \rightarrow$ Biomasa $\mathrm{CH}_{1,8} \mathrm{~N}_{0,5} \mathrm{O}_{0,2}+2 \mathrm{C}_{2} \mathrm{H}_{6} \mathrm{O}+2 \mathrm{CO}_{2}+\mathrm{H}_{2} \mathrm{O}$} \\
\hline & Fórmula & Grado de reducción & Reemplazando & Resultado \\
\hline $\begin{array}{l}\text { Sustrato } \\
\text { Sacarosa }\end{array}$ & $\begin{array}{c}\mathrm{C}_{\mathrm{w}} \mathrm{H}_{\mathrm{x}} \mathrm{O}_{\mathrm{y}} \\
\mathrm{C}_{12} \mathrm{H}_{22} \mathrm{O}_{11}\end{array}$ & $Y_{\text {sust }}=\frac{(4) \mathrm{w}+(1) \mathrm{x}+(-2) \mathrm{y}}{w}$ & $Y_{\text {sust }}=\frac{(4) 12+(1) 22+(-2) 11}{12}$ & Ysustrato $=4$ \\
\hline $\begin{array}{c}\text { Producto } \\
\text { Etanol }\end{array}$ & $\begin{array}{l}\mathrm{C}_{\mathrm{j}} \mathrm{H}_{\mathrm{y}} \mathrm{O}_{2} \\
\mathrm{C}_{2} \mathrm{H}_{6} \mathrm{O}_{1}\end{array}$ & $Y_{\text {Pro }}=\frac{(4) \mathrm{j}+(1) \mathrm{y}+(-2) \mathrm{z}}{j}$ & $Y_{\text {Pro }}=\frac{(4) 2+(1) 6+(-2) 1}{2}$ & YProducto $=6$ \\
\hline \multicolumn{5}{|c|}{ Rendimiento máximo de producto (Etanol) respecto al sustrato (Sacarosa) } \\
\hline$f_{\text {máx }}=\frac{u}{j}$ & $\frac{Y_{\text {Sustrato }}}{\text { Producto }}$ & $f_{\text {máx }}=\frac{12 * 4}{2 * 6}=4 \frac{\text { gmol producto }}{\text { gmol sustrato }}$ & $\begin{array}{r}Y_{p s}=4 \frac{\text { gmol producto }}{\text { gmol sustrato }} \times\left(\frac{46}{342,2}\right. \\
=0,5375 \mathrm{~g} / \mathrm{g}\end{array}$ & $\frac{\text { de etanol }}{\text { de glucosa }}$ \\
\hline
\end{tabular}

Tabla 6.

Rendimientos experimentales y máximos del proceso de fermentación acética

\begin{tabular}{|c|c|c|c|c|c|}
\hline & Fórmula & Grado de reducción & & Reemplazando & Resultado \\
\hline $\begin{array}{l}\text { Sustrato } \\
\text { Etanol }\end{array}$ & $\begin{array}{l}\mathrm{C}_{\mathrm{j}} \mathrm{H}_{\mathrm{y}} \mathrm{O}_{2} \\
\mathrm{C}_{2} \mathrm{H}_{6} \mathrm{O}_{1}\end{array}$ & $Y_{\text {sust }}=\frac{(4) j+(1) \gamma+(-2) z}{j}$ & $Y_{\text {sust }}$ & $=\frac{(4) 2+(1) 6+(-2) 1}{2}$ & $Y_{\text {sust }}=6$ \\
\hline $\begin{array}{l}\text { Producto } \\
\text { Ácido } \\
\text { acético }\end{array}$ & $\begin{array}{l}\mathrm{C}_{w} \mathrm{H}_{\mathrm{x}} \mathrm{O}_{\mathrm{y}} \\
\mathrm{C}_{2} \mathrm{H}_{4} \mathrm{O}_{2}\end{array}$ & $Y_{\text {Pro }}=\frac{(4) \mathrm{w}+(1) \mathrm{x}+(-2) \mathrm{y}}{w}$ & $Y_{\text {Pro }}=$ & $=\frac{(4) 2+(1) 4+(-2) 2}{2}$ & $\mathrm{YPro}_{\mathrm{P}}=4$ \\
\hline $\begin{array}{l}\text { Biomasa } \\
\text { Acetobacter } \\
\text { Acétic }\end{array}$ & $\begin{array}{c}\mathrm{C}_{w} \mathrm{H}_{\mathrm{x}} \mathrm{O}_{\mathrm{y}} \mathrm{N}_{z} \\
\mathrm{CH}_{1,4} \mathrm{O}_{0,4} \mathrm{~N}_{0,2}\end{array}$ & $\begin{array}{l}Y_{\text {biom }} \\
=\frac{(4) \mathrm{w}+(1) \mathrm{x}(-2) \mathrm{y}+(-3) \mathrm{z}}{j}\end{array}$ & $\begin{array}{l}Y_{\text {biom }} \\
= \\
=\end{array}$ & $\frac{1+(1) 1.4+(-2) 0.4-(3) 0.2}{2}$ & $Y_{\text {Biom }}=4$ \\
\hline \multicolumn{6}{|c|}{ Rendimiento teórico máximo de ácido acético respecto al sustrato (Alcohol): } \\
\hline \multicolumn{2}{|c|}{$f_{\text {máx }}=\frac{\boldsymbol{w} * \gamma_{\text {Sustrato }}}{j * \gamma_{\text {Producto }}}$} & \multicolumn{2}{|c|}{$f_{\text {máx }}=\frac{2 * 6}{4 * 2}=1,5 \frac{\text { gmol producto }}{\text { gmol sustrato }}$} & \multicolumn{2}{|c|}{$\therefore Y_{\text {ps máx }}=1,95 \frac{\mathrm{g} \text { producto }}{\mathrm{g} \text { sustrato }}$} \\
\hline \multicolumn{6}{|c|}{ Producción teórica máxima de biomasa respecto al sustrato (Alcohol) } \\
\hline$C_{\text {máx }}$ & $\frac{\boldsymbol{w} * \gamma_{\text {Sustrato }}}{\gamma_{\text {biomasa }}}$ & $C_{\text {máx }}=\frac{2 * 6}{4}=3 \frac{\text { gmolbion }}{\text { gmol sust }}$ & $\frac{\text { zasa }}{\text { rato }}$ & $\therefore Y_{x s \text { máx }}=1,4739 \frac{g b i}{g s u}$ & $\frac{\text { nasa }}{\text { rato }}$ \\
\hline
\end{tabular}


Cabe señalar que se obtuvo un rendimiento un Yps real de 1,0137 g producto/g sustrato. A partir de los rendimientos obtenidos a nivel experimental (tablas 5 y 6 ) se puede generar un balance de materia del proceso de elaboración del vinagre de vino, obteniendo un producto cuya concentración es de 18,8 g ácido acético/L.

Tabla 7.

Resultados del balance de materiales

En $450 \mathrm{ml}$ durante 4 días (108 h)

\begin{tabular}{|l|c|c|}
\hline Compuestos & \multicolumn{1}{c|}{ mol } & mol \\
\hline Etanol & 7,9901 & 0,1734 \\
\hline $\mathrm{O}_{2}$ (consumido) & 7,5947 & 0,2373 \\
\hline Fuente de N & 0,045 & 0,0026 \\
\hline Biomasa & 0,2986 & 0,0132 \\
\hline $\mathrm{CO}_{2}$ & 2,812 & 0,0639 \\
\hline $\mathrm{CH}_{3} \mathrm{COOH}$ & 8,1 & 0,1348 \\
\hline $\mathrm{H}_{2} \mathrm{O}$ & 4,4184 & 0,2452 \\
\hline \multicolumn{2}{|c|}{$\therefore$ Y $_{\text {ps real }}=\mathbf{1 , ~ 0 1 3 7 ~} \frac{\text { g producto }}{\boldsymbol{g} \text { sustrato }}$} \\
\hline
\end{tabular}

\subsection{Análisis fisicoquímico del vinagre del vino de cacao}

En primera instancia y sin ninguna corrección en el producto obtenido se cumplieron 4 de los 5 parámetros ya descritos en la norma INEN 2296, grado alcohólico, $\mathrm{pH}$, acidez fija, y acidez volátil, como se observa en la tabla 8. Esto hace necesaria la existencia de una etapa posterior en el proceso, debido a la alta concentración de ácido acético obtenido, siendo necesario añadir agua pura para disminuir su concentración y así obtener un vinagre que se encuentre en pleno cumplimiento de las especificaciones planteadas en la norma INEN.

Tabla 8.

Características físico-químicas del vinagre

\begin{tabular}{|l|c|}
\hline \multicolumn{1}{|c|}{ Muestra } & Resultados \\
\hline $\begin{array}{l}\text { Grado Alcohólico a 20 } \\
{ }^{\circ} \mathrm{C},\left({ }^{\circ} \mathrm{GL}\right)\end{array}$ & 0 \\
\hline $\begin{array}{l}\text { Acidez Total (ácido } \\
\text { acético) }(\mathrm{g} / \mathrm{L})\end{array}$ & 18,8 \\
\hline $\mathrm{pH}$ a $20{ }^{\circ} \mathrm{C}$ & 2,5 \\
\hline $\begin{array}{l}\text { Acidez fija, (como } \\
\text { ácido acético), \% } \mathrm{m} / \mathrm{v}\end{array}$ & 1,8451 \\
\hline $\begin{array}{l}\text { Acidez volátil, (como } \\
\text { ácido acético), \% m/v }\end{array}$ & 0,0249 \\
\hline
\end{tabular}

En otros proyectos dirigidos a la producción de vinagre de mucílago y exudado de cacao (Villagómez, 2013) se obtuvo un producto con acidez del 3,5 g/L para 24 horas de proceso de acetificación usando un cultivo madre de vino blanco cepa Chardonnay Blanc marca "Doña Dominga", mientras que en el proceso que se desarrolló en el presente trabajo al acetificar por 108 horas con una madre de vinagre 
obtenida a partir de las bacterias nativas del mucílago y exudado de cacao se logró una acidez total de $18,8 \mathrm{~g} / \mathrm{L}$, que pese al tiempo sigue siendo un rendimiento mucho más alto, aun así este factor debe ser analizado con mayor profundidad para efectos de producción masiva y costes económicos.

Según el Manual de Bebidas Alcohólicas y Vinagres (1999) el vino se ha transformado en vinagre cuando su contenido de alcohol se ha reducido a $1^{\circ} \mathrm{GL}$, sin embargo, al determinar el grado alcohólico en el vinagre obtenido, se constató que carecía de grado alcohólico, lo que es aún mejor, pues la norma establece como límite superior $1^{\circ} \mathrm{GL}$.

\section{Conclusiones}

Analógicamente, se conoce que el vinagre puede producirse directamente en un mismo proceso fermentativo, sin embargo, la implementación de la doble fermentación fue clave para el control de formación de sustrato secundario como el vino (etanol), que a su vez formaría una determinada cantidad de ácido acético, así como para fines analíticos, facilitando los procedimientos de medición y el cálculo de gases, biomasa y agua formados. Esto permitió realizar un estudio más profundo de los procesos de fermentación espontánea y evidentemente permitió mejorar las condiciones organolépticas del producto final, otorgándole un olor y sabor característicos del vinagre de vino.

Se determinó la cantidad óptima de solución de levadura en el mosto y la fuente de nitrógeno para bacterias acéticas; éstas permitieron acelerar y potenciar la fermentación natural que existe en el exudado. Por otra parte, la producción depende también de las propiedades reológicas, que en este caso están asociadas con el diámetro de partícula; en este sentido, es imperioso disponer de diámetros inferiores a $100 \mathrm{pm}$. Con mayor precisión, se recomienda que el tamaño de partícula más adecuado para permitir procesos óptimos de fluidez promedia las 25 ppm.

Además, se logró elevar en un $36 \%$ el aprovechamiento del fruto, al producir vinagre a partir del mucílago y exudado normalmente desechados, obteniendo un aprovechamiento parcial de los productos agrícolas en el Ecuador y 
paleando consecuentemente la contaminación ambiental que estos subproductos representan.

\section{Bibliografía}

AOAC. (1965). Official Methods of Analysis of the Association of Official Agricultural Chemists (10th ed. 1965). A.O.A.C., P.O.B. 540 Benjamin Franklin Station. Washington 4. D.C.

Braudeau, J. (2001). El Cacao: Técnicas Agrícolas $y$ Productos Tropicales. Barcelona-España: Editorial Blume.

Estrella, Y. A. (2013). Estudio del desperdicio del mucilago de cacao en el cantón Naranjal (Provincia del Guayas). ECA sinergia, 4(1), 49-59.

Feldsine, P., Abeyta, C., \& Andrews, W. H. (2002). AOAC International methods committee guidelines for validation of qualitative and quantitative food microbiological official methods of analysis. Journal of AOAC International, 85(5), 1187-1200.
INEN. (1978). NTE INEN 341. Bebidas alcohólicas. Determinación de acidez. Revisión. 1978-03.

INEN. (1978). NTE INEN 360. Bebidas alcohólicas. Determinación del grado alcohólico en vinos. Revisión. 1978-04.

INEN. (2013). NTE INEN 2296. Vinagre. Requisitos. Segunda revisión. 2013-04.

Llaguno, C., \& Polo, M. C. (1991). La composición química de los vinagres vínicos. El Vinagre de Vino. MC Eds., 105-132.

Molina, R. (2000). Teoría de la clarificación de mostos y vinos y sus aplicaciones prácticas (No. 663.2 M65).

Villagómez, S. (2013). Optimización y aprovechamiento del residuo (exudado del mucílago de la almendra fresca del caco (Theobroma cacao L.) CCN51 en la elaboración de vinagre (Bachelor's thesis, Universidad Tecnológica Equinoccial. Facultad: Ciencias de la Ingeniería). 